\title{
Analysis of Fetal Heart Rate Asymmetry before and after 35 weeks of gestation
}

\author{
Chandan Karmakar $^{1,2 *}$, Yoshitaka Kimura ${ }^{3}$, Marimuthu Palaniswami ${ }^{1}$ \\ and Ahsan Khandoker ${ }^{1,4}$
}

${ }^{1}$ Department of Electrical \& Electronic Engineering, University of Melbourne, Melbourne, Australia

${ }^{2}$ Centre for Pattern Recognition and Data Analytics, Deakin University, Geelong, Australia

${ }^{3}$ Graduate School of Medicine, Tohoku University, Sendai, Japan

${ }^{4}$ Department of Biomedical Engineering, Khalifa University of Science, Technology and Research, Abu Dhabi, UAE

Email: karmakar@unimelb.edu.au; ykimura@med.tohoku.ac.jp; palani@unimelb.edu.au;

ahsank@unimelb.edu.au;

\section{Address of corresponding author}

Department of Electrical \& Electronic Engineering

University of Melbourne

Parkville, VIC 3010

Melbourne, Australia

Email: karmakar@unimelb.edu.au

Phone: +61-3-90357614 
Abstract - In this study, we have investigated the evidence of fetal heart rate asymmetry and how the fetal heart rate asymmetry changes before and after 35 weeks of gestation. Noninvasive fetal electrocardiogram (fECG) signals from 45 pregnant women at the gestational age from16 to 41 weeks with normal single pregnancies were analysed. A nonlinear parameter called heart rate asymmetry (HRA) index that measures time asymmetry of RR interval time-series signal was used to understand the changes of HRA in early and late fetus groups. Results indicate that fetal HRA measured by Porta's Index (PI) consistently increases after 35 weeks gestation compared to foetus before 32 weeks of gestation. It might be due to significant changes of sympatho-vagal balance towards delivery with more sympathetic surge. On the other hand, Guzik's Index (GI) showed a mixed effect i.e., increases at lower lags and decreases at higher lags. Finally, fHRA could potentially help identify normal and the pathological autonomic nervous system development.

\section{Introduction}

Evaluation of fetal status is important for reducing perinatal morbidity and mortality. Advancements of science and technology promises to elucidate information regarding developmental changes during antenatal period. Thus, fetal death remains a potentially preventable condition that has major personal, social and financial implications for both families and the broader community.

Fetal well-being during pregnancy has been widely evaluated with fluctuations of fetal heart rate or fetal heart rate variability (fHRV) monitoring. This had started with introduction of cardiotocography (CTG - recording of fetal heart rate and force/pressure of contractions). However, initially it did not show any improvement over delivery outcomes [1] and became the main suspect for increased rate of cesarean sections [2]. After introducing the CTG interpretation guidelines in order to lower the number of neonates with asphyxia and cesarean sections [3, 4], it remains the most prevalent method for fetal surveillance $[5,6]$. Besides poor standard of interpretation and high intra/inter observer variability [2], CTG delivers only the momentary heart rate with limited accuracy [7]. Therefore, CTG derived fetal heart rate time-series is not suitable for beat-to-beat analysis, which is clinically more significant than long-term measure [8, 9]. Fetal magnetocardiogram (fMCG) overcomes these limitations of CTG and provides a non-invasive approach for monitoring fetal cardiac activity with sufficient precision [10, 11]. However, fMCG requires magnetically shielded rooms (MSR), which limits the regular use of the device due to the 
high cost of MSR materials. Like fMCG, fetal electrocardiogram (fECG) is a non-invasive method for monitoring beat-beat fetal cardiac activity with sufficient precision. Although, extraction of fECG is difficult, we have reported blind source separation with reference signals (BSSR) technique for stable and reliable extraction of fECG [12-15]. Therefore, fECG is the simple and convenient method for non-invasive monitoring of fetal cardiac activity.

The understanding of fetal neurological development in utero may lead to a better antenatal prediction of risk for adverse neurological outcomes, irrespective of intrapartum management. Since the fetal cardiac rhythm is controlled by the autonomic nervous system (ANS), the fHRV analysis is a simple way to understand the progressive development of ANS with gestational age (GA). At early GA, fetal heart rate is predominantly controlled by sympathetic nervous system (SNS) and arterial chemoreceptors [16]. With GA parasympathetic nervous system (PNS) matures and fetal heart rate (fHR) reduces with increasing variability [17]. In the normal fetus, interplay between SNS and PNS (ANS activity) controls the fHR and results in a difference in the beat-tobeat intervals resulting in variability of fHR tracing. This control mechanism is exerted via the cerebral cortex, the medulla oblongata, the sympathetic ganglia and the vagus nerve. The completion of the 35 gestational week marks a developmental milestone because of its association with a dramatically decreasing risk for the neonate in the case of a preterm delivery. About $90 \%$ of babies born this week can survive and lungs are almost fully developed.

Initial evaluation of fHRV was primarily based on the CTG assessment guidelines [3]. However, other methods derived from adult heart rate variability were used for fHRV analysis [18-21]. Since recent studies [22-25] have demonstrated that the recommendation standards for adult heart rate variability analysis [26] cannot be directly applied to fHRV studies, new linear and nonlinear parameters are started to be tested to analyze growth of fetus, classifying abnormal fHR and fetal status etc. [27-36]. Mostly used nonlinear methods are fractal dimension [18, $19,27]$ and entropy based regularity measurements (approximate entropy and sample entropy) [20, 28, 29, 31]. Recently we have used complex correlation measure (CCM), which measures the variability in the temporal structure of Poincaré, along with standard descriptors (SD1 and SD2) to investigate the changes in dynamics and variability of fHRV time-series with GA [37].

Nonlinear methods of HRV analysis have become prominent, since they have improved predictive power for determination of sudden cardiac death risk and characterization of disease states associated with autonomic nervous system function [38]. Heart rate asymmetry (HRA) is a Poincaré plot based nonlinear parameter for assessing time 
irreversibility of heart rate or RR interval time-series signal. Asymmetry analysis can detect a more specific type of nonlinear dynamics [39] capable of producing temporal asymmetries of the heart rate and resulting in statistical properties that are different when the heart rate series are observed after time reversal. HRA is defined based on acceleration and deceleration of heart rate based on two consecutive heart rates or RR intervals [40, 41]. In this definition, any point of Poincaré plot is classified as part of increasing or decreasing cloud with respect to line of identity $(y=x)$. In our previous study, another definition was proposed for defining acceleration and deceleration clouds to quantify HRA based on two points of Poincaré plot [42]. Both definitions have been used with popular HRA indices namely Guzki's Index (GI), Porta's Index (PI) and Ehlers' Index (EI). HRA analysis has been reported to analyse asymmetric properties of short and long-term HRV [40, 42], altered parasympathetic nervous system activity [43], severity of apnea [44], blood pressure variability [45] and cardiovascular dynamics [46]. There is no study done to look at HRA in fetal heart rates.

Therefore, the aim of this study was to check whether HRA is present in fetal heart rates and then how HRA changes over the gestational progression.

\section{Data and Methods}

\subsection{Data}

Recording of the abdominal ECG signals from 78 pregnant women at the gestational age of 16 41 weeks with normal single pregnancies were collected from Tohoku University Hospital. To evaluate change of HRA with gestational age, we divided the fetuses into two groups G1 $(\in[20,32])$ and $\mathrm{G} 2(\in[35,41])$. Since development of sympathetic nervous system completes by 35 weeks of gestation $[15,47]$, which is also associated with a dramatically decreased risk for the neonate in the case of a preterm delivery, it was used to divide the fetuses into two groups. We kept a gap of 3 weeks of gestation age between two groups for reducing overlapping effect. Out of 28 fetuses 22 cases were after 35 weeks and 46 were before 32 weeks of gestational age. Summary demography of two groups are shown in Table 1.

Table 1: Grouping of subjects based on gestational age (GA)

\begin{tabular}{|c|c|c|}
\hline Group & $\begin{array}{c}\text { GA range } \\
\text { (weeks) }\end{array}$ & $\begin{array}{c}\text { GA (weeks) } \\
\text { Mean } \pm \text { SD }\end{array}$ \\
\hline G1 & 20 to 32 & $25.89 \pm 3.85$ \\
\hline G2 & 35 to 41 & $37.95 \pm 1.43$ \\
\hline
\end{tabular}


All recordings (each of 1 minute's length) were sampled at $1000 \mathrm{~Hz}$ with 16-bit resolution. The study protocol was approved by Tohoku University Institutional Review Board and written informed consent was obtained from all subjects. fECG traces were extracted using a method that combines cancellation of the mother's ECG signal and the blind source separation with reference (BSSR) as described in our earlier study [15]. Intervals between successive R waves of the QRS complex (i.e., R-R intervals in seconds) were calculated using the algorithm developed by Pan and Tompkins [48]. Once RR intervals were calculated, the ectopic beat was selected automatically based on a predefined heart rate range. For any RR interval, if the instantaneous heart rate is lesser than 100 beats/minute or higher than 240 beats/minute then the interval was marked as ectopic and removed from the RR interval time-series. After artifact removal the average length of RR interval time-series for 68 recordings was 142 beats.

\subsection{HRA and HRA Indices}

HRA is defined as the imbalanced distribution of points above and below the line of identity $\left(L_{i}\right)$ of the Poincare plot (Figure 1). Points above, below and on $L_{i}$ have the property $\Delta R R>0, \Delta R R<0$ and $\Delta R R=0$ respectively, where $\Delta R R=R R_{i+1}-R R_{i}$ and $R R_{i}$ represents the $i$-th $\mathrm{RR}$ interval.

\subsubsection{Guzik’s Index $(G I)$ :}

Guzik et al. have defined the index for measuring the asymmetry of the time series using a Poincaré plot [40]. GI is defined as the distance of the plotted points from the line of identity. Any point on the plot is given as $P_{i}\left(R R_{i}, R R_{i+1}\right)$. The distance from line of identity is calculated as:

$$
D_{i}=\frac{\left|R R_{i}-R R_{i+1}\right|}{\sqrt[2]{2}}
$$

and $G I$ is defined as:

$$
G I=\frac{\sum_{i=1}^{C\left(P_{i}^{+}\right)}\left(D_{i}^{+}\right)^{2}}{\sum_{i=1}^{N-1}\left(D_{i}\right)^{2}} \times 100
$$

where, $P_{i}^{+}$represents a point above the line of identity $\left(R R_{i}<R R_{i+1}\right), D_{i}^{+}$represents the corresponding distance of $P_{i}^{+}$from the line of identity, $C\left(P_{i}^{+}\right)$represents the total number of points above the line of identity and $N$ is the total number of RR intervals.

\subsubsection{Porta's Index $(P I)$ :}


In contrast to the distance from the line of identity, PI is defined based on the distribution of points below and above the line of identity. PI is calculated as the percentage of the number of points below the line of identity with respect to the total number of points [41].

$$
P I=\frac{C\left(P_{i}^{-}\right)}{C\left(P_{i}^{+}\right)+C\left(P_{i}^{-}\right)} \times 100
$$

where, $P_{i}^{-}$represents the point below the line of identity $\left(R R_{i}>R R_{i+1}\right), C\left(P_{i}^{-}\right)$and $C\left(P_{i}^{+}\right)$represent the total number of points below and above the line of identity respectively.

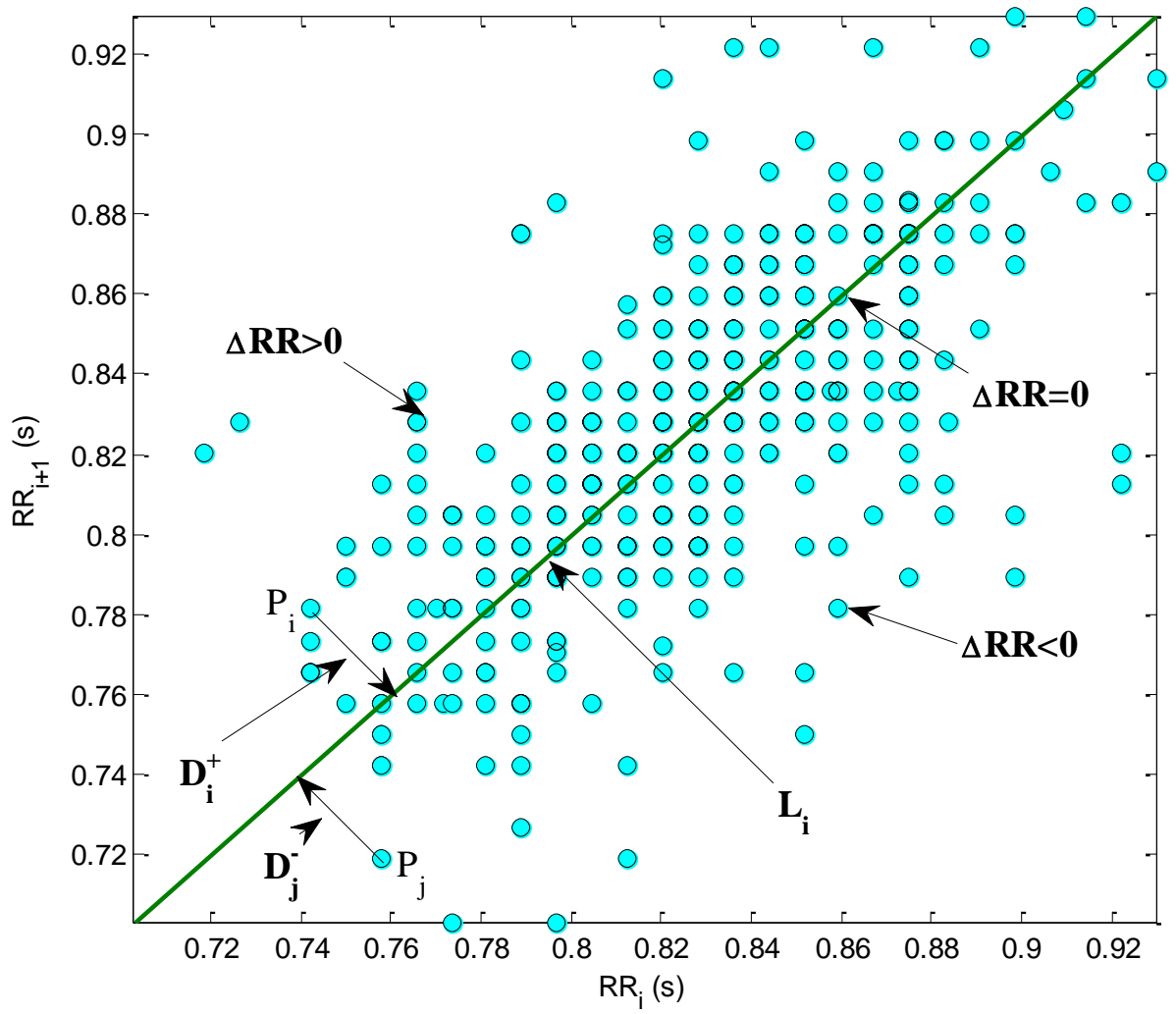

Figure 1: Properties of points in a lag-1 Poincaré plot used to calculate Guzik's and Porta's asymmetry indexes.

The following HRA indices were used to measure the asymmetry of the RR interval time-series:

\subsection{Multi-lag HRA}

Poincaré plot is a time-delayed map of time-series signal, where the time delay termed as lag of the plot. Therefore, lag is an inherent property of Poincaré plot. The conventional method of quantifying HRA uses successive RR intervals with the implicit assumption that the current beat is influenced by the immediately preceding beat. However, the delay of such influence is affected by the variation of baroreflex sensitivity and therefore can be greater than one beat $[49,50]$. For example, the baroreflex sensitivity is reduced in chronic renal failure (CRF) 
patients [51], which augments the delay in the heart rate response. Therefore, a heartbeat influences not only the beat immediately following it, but also up to 6-10 beats downstream [52] and several researchers confirmed that the lag is associated with disease processes [53, 54]. Thus, successive RR interval duplets will likely underestimate the role of the autocovariance function of RR intervals i.e., the ability of heart beats to influence a train of succeeding beats. Moreover, the autocovariance function of RR intervals captures additional aspects of HRV (e.g. non-linearity) that are otherwise masked by the strong correlation between successive beats if lag 1 ( $n$ vs $n+1$ beats) HRA is used. Therefore, we hypothesized that multi-lag HRA analysis will provide better understanding about the development of ANS in foetus with gestation.

For multi-lag HRA analysis, we have introduced the lag $(m)$ in equation $(2)$ and (3), used to calculate GI and PI from RR interval time series signal. In lag $-m$ Poincare plot the $i$-th point on the plot is the duplet $P_{i}^{m}\left(R R_{i}, R R_{i+m}\right)$. Hence, in the multi-lag HRA analysis GI and PI indices were calculated using following equations:

$$
\begin{gathered}
G I_{m}=\frac{\sum_{i=1}^{C\left(P_{i}^{m+}\right)}\left(D_{i}^{m+}\right)^{2}}{\sum_{i=1}^{N-1}\left(D_{i}^{m}\right)^{2}} \times 100 \\
P I=\frac{C\left(P_{i}^{m-}\right)}{C\left(P_{i}^{m+}\right)+C\left(P_{i}^{m-}\right)} \times 100
\end{gathered}
$$

where, $P_{i}^{m+}$ and $P_{i}^{m-}$ represent the point above and below line of identity at lag $m . C\left(P_{i}^{m+}\right)$ and $C\left(P_{i}^{m-}\right)$ represent the number of points below and above line of identity at lag $m . D_{i}^{m}$ represents the distance of point $P_{i}^{m}$ from line of identity. In this study, we have used lag $m=1$ to 8 for analyzing HRA of fetal RR interval timeseries signal.

\subsection{Statistics}

The non-parametric Mann-Whitney U-test was performed to allow pair-wise testing for significant differences of HRA parameters between the two groups G1 and G2. Since, the number of subjects is small and their distribution is not normal, which was tested using Lilliefors test, a non-parametric test is more appropriate than parametric test. For each group, we estimated the dependence of the HRA indices on fetal maturation by calculating their correlation with GA. The Spearman's rank-order correlation coefficient $r$ was calculated to measure the strength of the association between HRA index and gestation age. Spearman's rank correlation coefficient is a nonparametric (distribution-free) rank statistic, which measures the monotone association that is used when the distribution of data 
makes Pearson's correlation coefficient undesirable or misleading. It assesses how well an arbitrary monotonic function can describe the relationship between two variables, without making any assumptions about the frequency distribution of the variables. Besides strength of the correlation it also provides significance of the correlation measured as $p$. In this study, $p<0.05$ was considered statistically significant.
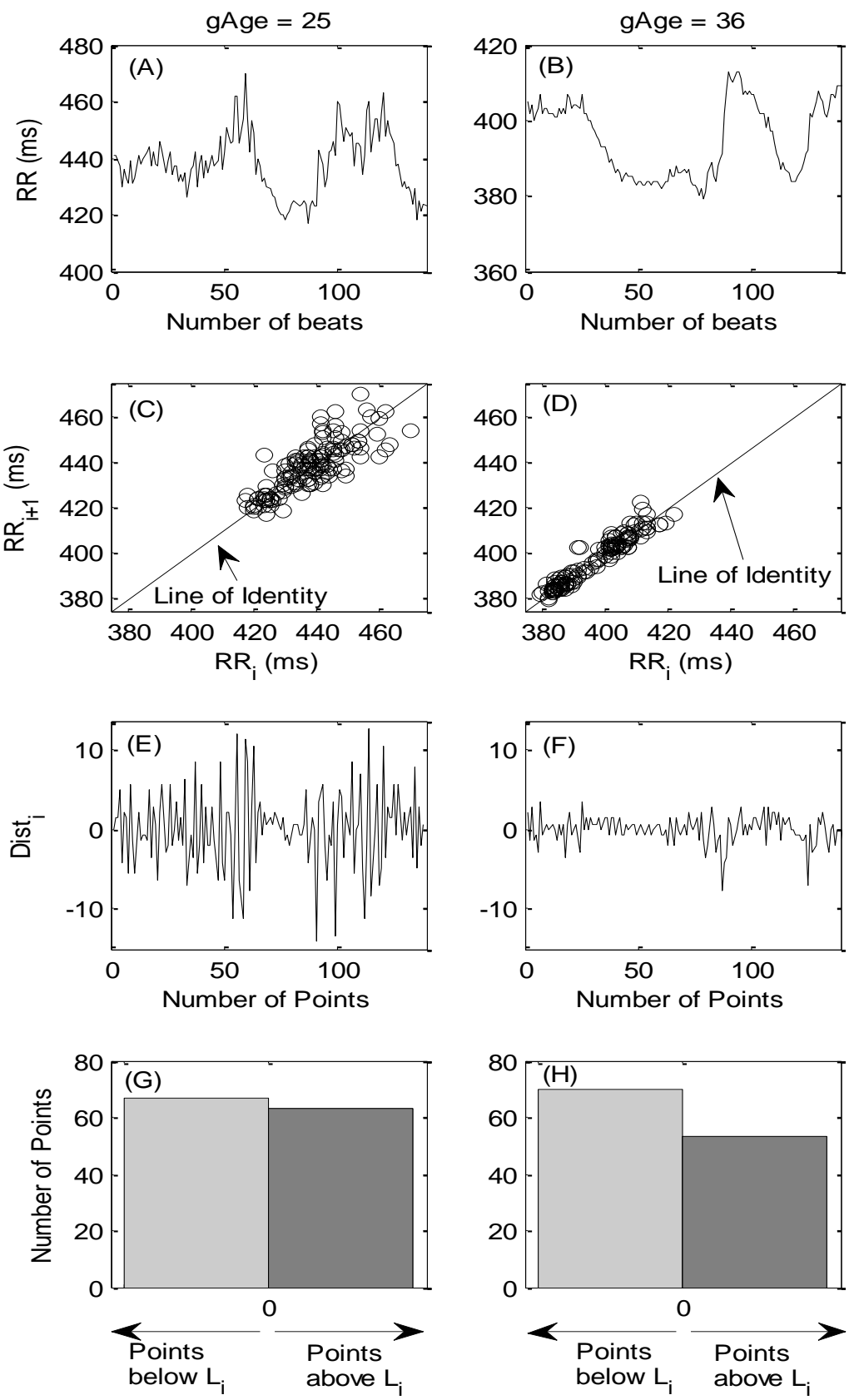

Figure 2: An example i) RR trace (A-B) of two groups; ii) corresponding lag - 1 Poincaré plot (C-D); iii) Distance from line of identity; and iv) Distribution of points below and above line of identity. 


\section{Results}

A typical example of RR interval traces of one subject of each group is shown in Figure 2 (A-B). The lag-1 Poincaré plots corresponding to the RR intervals are shown in subplots C-D. The distance $D_{i}$ of each point of the plot is shown in subplots E-F. The HRA index GI is calculated from these distances using equation 2. Subplots G$\mathrm{H}$ represents the distribution of number of points above and below line of identity corresponding to Poincaré plots of subplots C-D. Figure 2 provides a complete visualization of how HRA indices are calculated from RR interval time series signal.

The mean \pm SD (standard deviation) values of HRA indices for each group are shown in Table 2 and the corresponding error bar plot is shown in Figure 3. Mean GI values were higher in early gestation age group (G1) than late gestation age group (G2) at all lags $m=1$ to 8 . Although mean $G I$ values were higher in $\mathrm{G} 1$ than $\mathrm{G} 2$ at all lags, the difference was statistically not significant ( $\mathrm{p}>0.05$ ). In contrast, $P I$ values were higher in G2 than G1 at all lags and the difference was statistically significant $(\mathrm{p}<0.05)$ for lags $m=1$ to 4 .

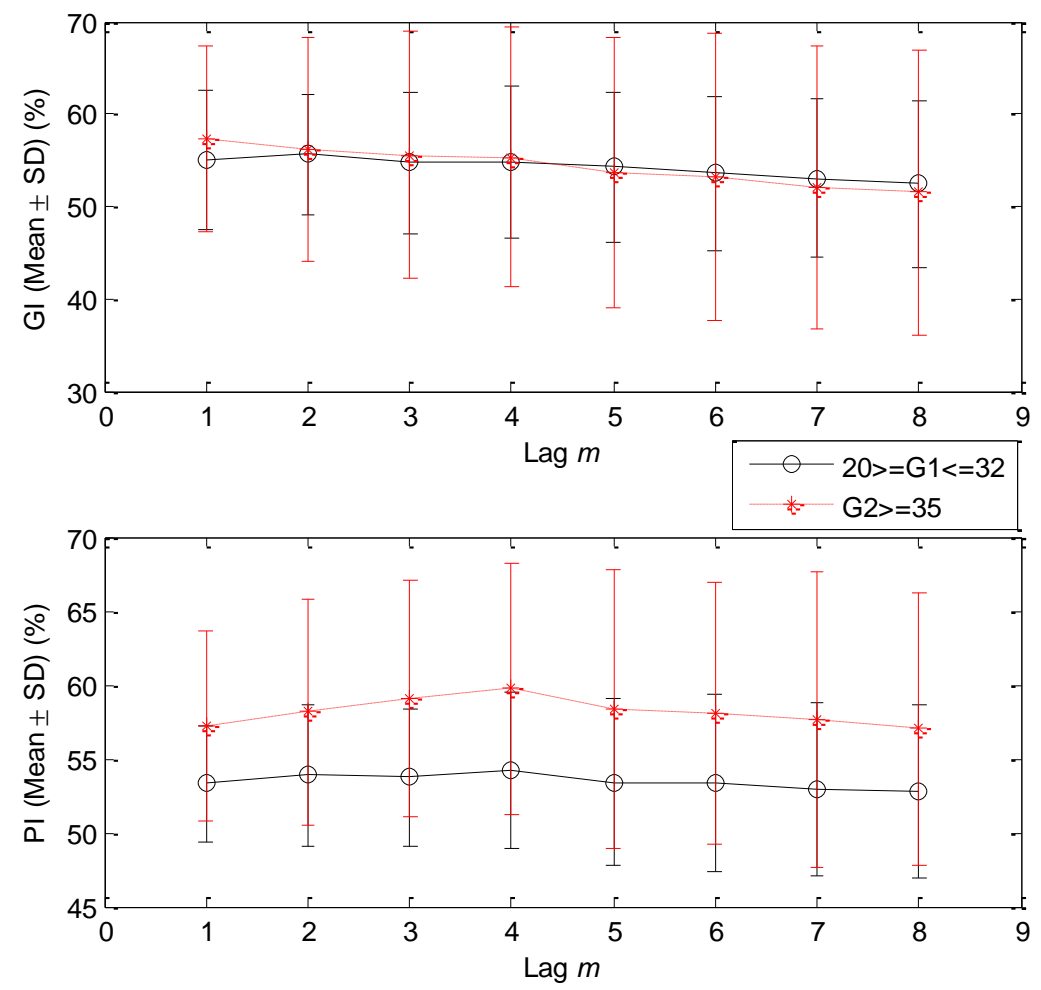

Figure 3: Errorbar (Mean $\pm \mathrm{SD}$ ) of HRA indices GI and PI at lags $m=1 \sim 8$. 
Table 2. Mean \pm SD values of HRA parameters (GI and PI) at lags $m=1 \sim 8$. $p$ values were calculated using Mann-Whitney $\mathrm{U}$ test. * indicates values between two groups is significant $(p<0.05)$.

\begin{tabular}{|c|r|c|c|c|}
\hline \multirow{3}{*}{ Parameter } & $\begin{array}{c}\text { Lag } \\
\mathrm{m}\end{array}$ & $\begin{array}{c}\mathrm{G} 1 \\
\text { Mean } \pm \text { SD }\end{array}$ & $\begin{array}{c}\text { G2 } \\
\text { Mean } \pm \text { SD }\end{array}$ & $p$ \\
\hline \multirow{7}{*}{ GI } & 1 & $55.05 \pm 7.54$ & $57.37 \pm 10.02$ & 0.508 \\
\cline { 2 - 5 } & 2 & $55.60 \pm 6.49$ & $56.17 \pm 12.16$ & 0.516 \\
\cline { 2 - 5 } & 3 & $54.70 \pm 7.68$ & $55.54 \pm 13.41$ & 0.335 \\
\cline { 2 - 5 } & 4 & $54.76 \pm 8.16$ & $55.32 \pm 14.06$ & 0.246 \\
\cline { 2 - 5 } & 5 & $54.24 \pm 8.05$ & $53.59 \pm 14.63$ & 0.420 \\
\cline { 2 - 5 } & 6 & $53.58 \pm 8.38$ & $53.18 \pm 15.46$ & 0.362 \\
\cline { 2 - 5 } & 7 & $53.04 \pm 8.57$ & $52.10 \pm 15.31$ & 0.459 \\
\cline { 2 - 5 } & 8 & $52.44 \pm 8.98$ & $51.57 \pm 15.43$ & 0.467 \\
\hline \multirow{7}{*}{ PI } & 1 & $53.29 \pm 3.88$ & $57.21 \pm 6.49$ & $0.006^{*}$ \\
\cline { 2 - 5 } & 2 & $53.86 \pm 4.75$ & $58.15 \pm 7.65$ & $0.014^{*}$ \\
\cline { 2 - 5 } & 3 & $53.72 \pm 4.58$ & $59.07 \pm 8.06$ & $0.008^{*}$ \\
\cline { 2 - 5 } & 4 & $54.24 \pm 5.32$ & $59.71 \pm 8.56$ & $0.009^{*}$ \\
\cline { 2 - 5 } & 5 & $53.39 \pm 5.63$ & $58.39 \pm 9.45$ & 0.060 \\
\cline { 2 - 5 } & 6 & $53.36 \pm 5.93$ & $58.08 \pm 8.89$ & 0.064 \\
\cline { 2 - 5 } & 7 & $52.92 \pm 5.83$ & $57.62 \pm 10.02$ & 0.111 \\
\cline { 2 - 5 } & 8 & $52.76 \pm 5.86$ & $57.01 \pm 9.17$ & 0.114 \\
\hline
\end{tabular}
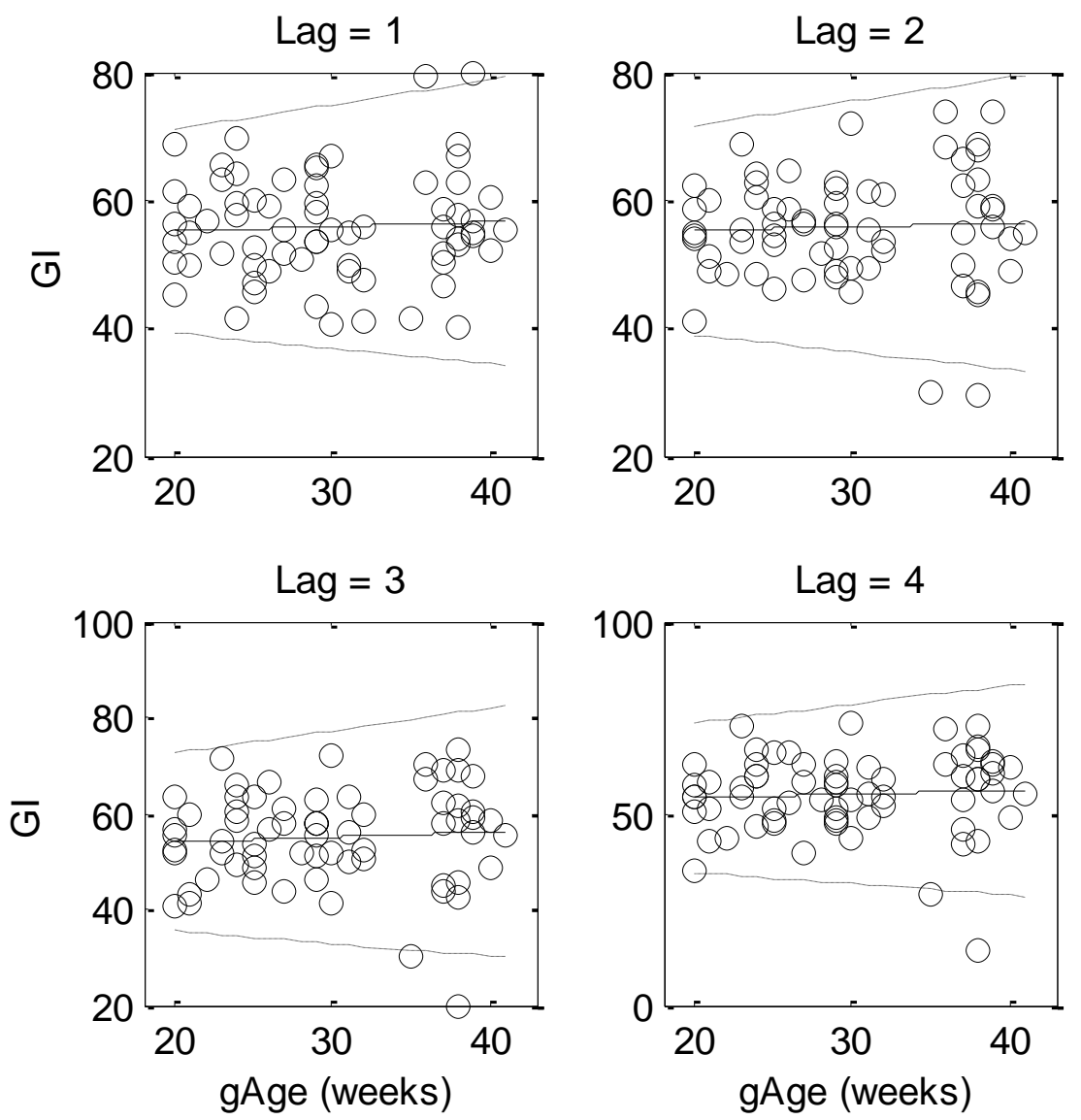

Figure 4: Correlation between GI and gestational age at lags $\mathrm{m}=1 \sim 4$. 

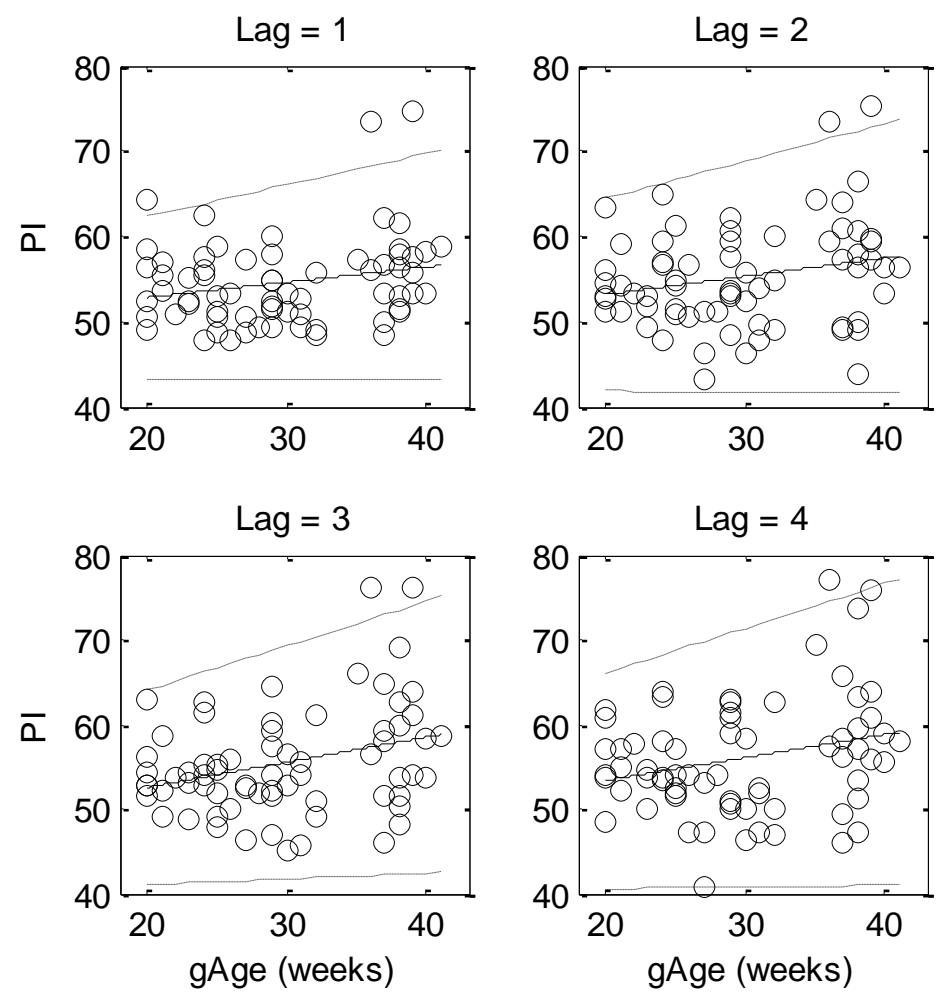

Figure 5: Correlation between PI and gestational age at lags $m=1 \sim 4$.

Table 3: Spearman correlation coefficient $(\mathrm{R})$ and corresponding $\mathrm{p}$ values for all lags.

\begin{tabular}{|c|r|c|c|}
\hline Parameter & Lag $m$ & $\mathrm{R}$ & $P$ \\
\hline \multirow{7}{*}{ GI } & 1 & 0.06 & 0.65 \\
\cline { 2 - 4 } & 2 & 0.04 & 0.75 \\
\cline { 2 - 4 } & 3 & 0.07 & 0.57 \\
\cline { 2 - 4 } & 4 & 0.06 & 0.61 \\
\cline { 2 - 4 } & 5 & 0.00 & 0.99 \\
\cline { 2 - 4 } & 6 & 0.04 & 0.77 \\
\cline { 2 - 4 } & 7 & 0.04 & 0.77 \\
\cline { 2 - 4 } & 8 & 0.04 & 0.72 \\
\hline \multirow{7}{*}{ PI } & 1 & 0.23 & 0.06 \\
\cline { 2 - 4 } & 2 & 0.23 & 0.06 \\
\cline { 2 - 4 } & 3 & 0.30 & 0.01 \\
\cline { 2 - 4 } & 4 & 0.26 & 0.03 \\
\cline { 2 - 4 } & 5 & 0.22 & 0.07 \\
\cline { 2 - 4 } & 6 & 0.23 & 0.06 \\
\cline { 2 - 4 } & 7 & 0.24 & 0.05 \\
\cline { 2 - 4 } & 8 & 0.22 & 0.07 \\
\hline
\end{tabular}

The relationships of $G I$ and $P I$ at multiple lags (1 4) with gestational ages are illustrated in Figures 4 and 5 . GI was increasing with gestational ages however the relationships were statistically not significant $(p>0.05$ in 
Table 3). Similar to GI, PI was also increasing with gestational ages and significant $(p<0.05)$ correlations were only seen at lags 3 and 4 (Table 3).

\section{Discussion}

The goal of this study was to contribute to the assessment of the effectiveness of fHRV measures in further understanding the prenatal development of integrative ANS function prior to 32 and after 35 weeks of gestation, employing Poincaré plot based heart rate asymmetry analysis. Various linear and nonlinear analysis of fHRV have been applied for the classification of abnormal and compromised foetus $[55,56]$. These applications are encouraged by the findings that recommendations of standard for adult HRV analysis are not directly applicable to fHRV [22-25]. However, study of HRA in prenatal development has never been reported before to our knowledge. Poincaré plot technique can be applied on short-term data to explore the variability and dynamics in time domain as previously applied in other studies $[4,14]$. Fetal electrocardiography (ECG) that provides superior measurement of beat-to-beat intervals was used in this study. For many of the current HRV analysis methods the assumption is that for all consecutive beats, each beat only has influence on the subsequent beat, whereas it has been shown that each beat in a time series can have an influence on up to ten beats downstream [52]. This opened up the avenue for analyzing multi-lag HRA characteristics of beat to beat fetal heart rate time series and its usefulness in clinical application.

There are various measures available to quantify asymmetry in cardiovascular signals. Recent studies suggest that simple HRA indices such as Guzik's and Porta's index are sensitive to shifts in sympatho-vagal balance. PI compares the number of increments and decrements in the RR time-series signal (i.e. deceleration and acceleration of the heart rate), whereas GI compares the magnitude of such acceleration and deceleration. These different methods may provide partially independent information and their simultaneous quantification might be useful in order to detect asymmetry more comprehensively.

Within the context of fetal development with gestational age the current results highlight that the asymmetry of the number of accelerations and decelerations (measured by PI), that is, sympathetic and parasympathetic influence changed significantly at late GA for lags $m=1$ to 4 . However, the asymmetry of the magnitude of these accelerations/decelerations (measured by GI) remained similar in both groups for all lags. Therefore, the results showed that there is a significant variation in number of acceleration and deceleration of RR intervals with 
gestation age rather than magnitude of such acceleration or deceleration.

PI values greater than 50\% indicate that there are higher number HR acceleration points (point below line of identity using RR intervals) than deceleration. Therefore, higher values of PI in G2 (gestation age greater than 35) suggest that there are more numbers of instant heart rate increments in this group than G1. Since increase in HR is linked with increased sympathetic activity, the increased value of PI in G2 may signify the maturation of sympathetic nervous system. This findings is supported by a histologic study of the cardiac conduction system in human fetal hearts, which reported that the sinus node is completely developed by the 6th gestational week, the atrioventricular node by the 10th gestational week, and the entire functional conduction system by the 16th gestational week [57]. However, development of the sympathetic nerves begins around the 20th gestational week, later than does that of the parasympathetic nerves, and is most rapid during the 26th to 30th gestational weeks, which coincide with the period of rapid increase in the low frequency (LF) power [47].

The mean PI values of late foetus group were initially increased for lags $m=1$ to 4 and then decreased with increasing lags $m=5$ to 8 . This indicates that the sympathetic influence increases with increasing lags up to 4 and then decreases with further increasing in lag. On the other hand, in early foetus group mean PI consistently decreased with increasing lags $\mathrm{m}=1$ or 8 , which could mean that parasympathetic influence of current beat increases with increasing lag (distant heart beats) in early foetus group.

Both GI and PI showed positive correlation with gestation age, which indicates that maturation of autonomic nervous system increases fetal heart rate asymmetry. In contrast to PI, which showed significant correlation at lag 3 and 4, GI showed no significant correlation with gestation age. This indicates that the effect of gestation age is more pronounced in the asymmetricities of number of acceleration/deceleration rather than magnitude. Moreover, such relationship is strongly pronounced at lag 3 and 4 than lag 1 . Therefore, multi-lag analysis is required to better understand the fetal autonomic nervous system development with gestation using heart rate asymmetry analysis.

This study is limited by the small number of subjects and short length recordings. Although no study has been reported result of HRA on 1 minute ECG recordings, it has been applied on short-term analysis of 5 minute ECG recordings of healthy adult subjects $[40,42]$. Therefore, further study is required on large cohort with longer recordings to confirm the repeatability of the findings of the present study. 


\section{Conclusion}

In summary, a HRA index (Porta's Indices) of fHRV at multiple lags increases from early (before 32 weeks) to late (after 35 weeks). Increasing PI of late foetus group could be an alternate expression of shifted sympathovagal balance to an increased sympathetic activity. Fetal development causes an alteration in the HRA indices that correlates with the progressive innervations of sympathetic activity towards delivery.

\section{Acknowledgement}

This study was supported by an Australian Research Council (ARC) linkage project (LP100200184) with Atom

Medical Corporation, Japan.

\section{References}

[1] Z. Alfirevic, D. Devane, G. Gyte, Continuous cardiotocography (CTG) as a form of electronic fetal monitoring (EFM) for fetal assessment during labour, Cochrane Database Syst Rev, 3 (2006).

[2] P.J. Steer, Has electronic fetal heart rate monitoring made a difference?, in: Seminars in Fetal and Neonatal Medicine, Elsevier, 2008, pp. $2-7$.

[3] G. Healthcare, Electronic fetal heart rate monitoring: research guidelines for interpretation, J Obstet Gynecol Neonatal Nurs, 26 (1997) 635-640.

[4] G. Rooth, A. Huch, R. Huch, FIGO News: guidelines for the use of fetal monitoring, Int J Gynecol Obstet, 25 (1987) 159167.

[5] J. Bernardes, A. Costa-Pereira, D. Ayres-de-Campos, H. Van Geijn, L. Pereira-Leite, Evaluation of interobserver agreement of cardiotocograms, International Journal of Gynecology \& Obstetrics, 57 (1997) 33-37.

[6] O. Palomäki, T. Luukkaala, R. Luoto, R. Tuimala, Intrapartum cardiotocography-the dilemma of interpretational variation, J Perinat Med, 34 (2006) 298-302.

[7] G. Dawes, M. Moulden, C. Redman, Limitations of antenatal fetal heart rate monitors, American journal of obstetrics and gynecology, 162 (1990) 170-173.

[8] M. Druzin, Antepartum fetal heart rate monitoring. State of the art, Clinics in perinatology, 16 (1989) 627-642.

[9] E.F. Hamilton, P.A. Warrick, New perspectives in electronic fetal surveillance, J Perinat Med, 41 (2013) 83-92.

[10] Z. Dunajski, M. Peters, Development of the fetal magnetocardiograms from the 13th week of gestation onward, Stud Appl Electromag, 7 (1995) 704-708.

[11] A. Quinn, A. Weir, U. Shahani, R. Bain, P. Maas, G. Donaldson, Antenatal fetal magnetocardiography: a new method for fetal surveillance?, BJOG: An International Journal of Obstetrics \& Gynaecology, 101 (1994) 866-870.

[12] S. Furukawa, H. Sameshima, L. Yang, T. Ikenoue, Acetylcholine receptor agonist reduces brain damage induced by hypoxia-ischemia in newborn rats, Reproductive Sciences, 18 (2011) 172-179.

[13] Y. Kimura, K. Okamura, T. Watanabe, J. Murotsuki, T. Suzuki, M. Yano, A. Yajima, Power spectral analysis for autonomic influences heart rate and blood pressure variability in fetal, (1996).

[14] Y. Kimura, N. Sato, J. Sugawara, C. Velayo, T. Hoshiai, S. Nagase, T. Ito, Y. Onuma, A. Katsumata, K. Okamura, Recent Advances in Fetal Electrocardiography, Open Medical Devices Journal, 4 (2012) 7-12.

[15] M. Sato, Y. Kimura, S. Chida, T. Ito, N. Katayama, K. Okamura, M. Nakao, A novel extraction method of fetal electrocardiogram from the composite abdominal signal, Biomedical Engineering, IEEE Transactions on, 54 (2007) 49-58.

[16] P. Renou, W. Newman, C. Wood, Autonomic control of fetal heart rate, Am J Obstet Gynecol, 105 (1969) 949-953.

[17] R. Gagnon, K. Campbell, C. Hunse, J. Patrick, Patterns of human fetal heart rate accelerations from 26 weeks to term, American journal of obstetrics and gynecology, 157 (1987) 743-748.

[18] D.G. Chaffin, C.C. Goldberg, K.L. Reed, The dimension of chaos in the fetal heart rate, American journal of obstetrics and gynecology, 165 (1991) 1425-1429.

[19] N.A.J. Gough, Fractal analysis of foetal heart rate variability, Physiological measurement, 14 (1993) 309.

[20] S.M. Pincus, R.R. Viscarello, Approximate entropy: a regularity measure for fetal heart rate analysis, Obstetrics \& Gynecology, 79 (1992) 249-255.

[21] E. Salamalekis, P. Thomopoulos, D. Giannaris, I. Salloum, G. Vasios, A. Prentza, D. Koutsouris, Computerised intrapartum diagnosis of fetal hypoxia based on fetal heart rate monitoring and fetal pulse oximetry recordings utilising wavelet analysis and neural networks, BJOG: An International Journal of Obstetrics \& Gynaecology, 109 (2002) 1137-1142. 
[22] S. Lange, P. Van Leeuwen, D. Geue, W. Hatzmann, D. Grönemeyer, Influence of gestational age, heart rate, gender and time of day on fetal heart rate variability, Medical and Biological Engineering and Computing, 43 (2005) 481-486.

[23] S. Lange, P. Van Leeuwen, U. Schneider, B. Frank, D. Hoyer, D. Geue, D. Grönemeyer, Heart rate features in fetal behavioural states, Early human development, 85 (2009) 131-135.

[24] U. Schneider, B. Frank, A. Fiedler, C. Kaehler, D. Hoyer, M. Liehr, J. Haueisen, E. Schleussner, Human fetal heart rate variability-characteristics of autonomic regulation in the third trimester of gestation, J Perinat Med, 36 (2008) 433-441.

[25] U. Schneider, E. Schleussner, A. Fiedler, S. Jaekel, M. Liehr, J. Haueisen, D. Hoyer, Fetal heart rate variability reveals differential dynamics in the intrauterine development of the sympathetic and parasympathetic branches of the autonomic nervous system, Physiological measurement, 30 (2009) 215.

[26] A. Camm, M. Malik, J. Bigger, G. Breithardt, S. Cerutti, R. Cohen, P. Coumel, E. Fallen, H. Kennedy, R. Kleiger, Heart rate variability: standards of measurement, physiological interpretation and clinical use. Task Force of the European Society of Cardiology and the North American Society of Pacing and Electrophysiology, Circulation, 93 (1996) 1043-1065.

[27] C. Felgueiras, J.M. de Sá, J. Bernardes, S. Gama, Classification of foetal heart rate sequences based on fractal features, Medical and Biological Engineering and Computing, 36 (1998) 197-201.

[28] M. Ferrario, M.G. Signorini, G. Magenes, Complexity analysis of the fetal heart rate variability: early identification of severe intrauterine growth-restricted fetuses, Medical \& biological engineering \& computing, 47 (2009) 911-919.

[29] M. Ferrario, M.G. Signorini, G. Magenes, S. Cerutti, Comparison of entropy-based regularity estimators: application to the fetal heart rate signal for the identification of fetal distress, Biomedical Engineering, IEEE Transactions on, 53 (2006) $119-125$.

[30] G. Georgoulas, C. Stylios, P. Groumpos, Feature extraction and classification of fetal heart rate using wavelet analysis and support vector machines, International Journal on Artificial Intelligence Tools, 15 (2006) 411-432.

[31] H. Gonçalves, J. Bernardes, A. Paula Rocha, D. Ayres-de-Campos, Linear and nonlinear analysis of heart rate patterns associated with fetal behavioral states in the antepartum period, Early human development, 83 (2007) 585-591.

[32] H. Gonçalves, A.P. Rocha, D. Ayres-de-Campos, J. Bernardes, Linear and nonlinear fetal heart rate analysis of normal and acidemic fetuses in the minutes preceding delivery, Medical and Biological Engineering and Computing, 44 (2006) 847 855.

[33] A. Kikuchi, N. Unno, T. Horikoshi, T. Shimizu, S. Kozuma, Y. Taketani, Changes in fractal features of fetal heart rate during pregnancy, Early human development, 81 (2005) 655-661.

[34] M.G. Signorini, G. Magenes, S. Cerutti, D. Arduini, Linear and nonlinear parameters for the analysis of fetal heart rate signal from cardiotocographic recordings, Ieee T Bio-Med Eng, 50 (2003) 365-374.

[35] J. Van Laar, M. Porath, C. Peters, S. Oei, Spectral analysis of fetal heart rate variability for fetal surveillance: review of the literature, Acta obstetricia et gynecologica Scandinavica, 87 (2008) 300-306.

[36] P.A. Warrick, E.F. Hamilton, D. Precup, R.E. Kearney, Classification of normal and hypoxic fetuses from systems modeling of intrapartum cardiotocography, Biomedical Engineering, IEEE Transactions on, 57 (2010) 771-779.

[37] A.H. Khandoker, C. Karmakar, Y. Kimura, M. Palaniswami, Development of fetal heart rate dynamics before and after 30 and 35 weeks of gestation, in: Computing in Cardiology Conference (CinC), 2013, IEEE, 2013, pp. 453-456.

[38] A. Voss, J. Kurths, H. Kleiner, A. Witt, N. Wessel, P. Saparin, K. Osterziel, R. Schurath, R. Dietz, The application of methods of non-linear dynamics for the improved and predictive recognition of patients threatened by sudden cardiac death, Cardiovascular research, 31 (1996) 419-433.

[39] G. Weiss, Time-reversibility of linear stochastic processes, Journal of Applied Probability, (1975) 831-836.

[40] P. Guzik, J. Piskorski, T. Krauze, A. Wykretowicz, H. Wysocki, Heart rate asymmetry by Poincaré plots of RR intervals, Biomedizinische Technik, 51 (2006) 272-275.

[41] A. Porta, K.R. Casali, A.G. Casali, T. Gnecchi-Ruscone, E. Tobaldini, N. Montano, S. Lange, D. Geue, D. Cysarz, P. Van Leeuwen, Temporal asymmetries of short-term heart period variability are linked to autonomic regulation, American Journal of Physiology-Regulatory, Integrative and Comparative Physiology, 295 (2008) R550-R557.

[42] C. Karmakar, A. Khandoker, J. Gubbi, M. Palaniswami, Defining asymmetry in heart rate variability signals using a Poincaré plot, Physiological measurement, 30 (2009) 1227.

[43] C. Karmakar, A. Khandoker, M. Palaniswami, Investigating the changes in heart rate asymmetry (HRA) with perturbation of parasympathetic nervous system, Australasian Physical \& Engineering Sciences in Medicine, 35 (2012) 465 474.

[44] P. Guzik, J. Piskorski, K. Awan, T. Krauze, M. Fitzpatrick, A. Baranchuk, Obstructive sleep apnea and heart rate asymmetry microstructure during sleep, Clinical Autonomic Research, 23 (2013) 91-100.

[45] P. Guzik, J. Piskorski, T. Krauze, K. Narkiewicz, A. Wykretowicz, H. Wysocki, Asymmetric features of short-term blood pressure variability, Hypertension Research, 33 (2010) 1199-1205.

[46] P. Guzik, J. Piskorski, T. Krauze, A. Wykretowicz, H. Wysocki, Partitioning total heart rate variability, International journal of cardiology, 144 (2010) 138-139.

[47] T. Ohta, K. Okamura, Y. Kimura, T. Suzuki, T. Watanabe, T. Yasui, N. Yaegashi, A. Yajima, Alteration in the LowFrequency Domain in Power Spectral Analysisof Fetal Heart Beat Fluctuations, Fetal diagnosis and therapy, 14 (1999) 92-97.

[48] J. Pan, W.J. Tompkins, A Real-Time Qrs Detection Algorithm, Ieee T Bio-Med Eng, 32 (1985) 230-236. 
[49] S. Cavalcanti, E. Belardinelli, Modeling of cardiovascular variability using a differential delay equation, Biomedical Engineering, IEEE Transactions on, 43 (1996) 982-989.

[50] J.T. Ottesen, Modelling of the baroreflex-feedback mechanism with time-delay, Journal of mathematical biology, 36 (1997) 41-63.

[51] O. Tomiyama, T. Shiigai, T. Ideura, K. Tomita, Y. Mito, S. Shinohara, J. Takeuchi, Baroreflex sensitivity in renal failure, Clin Sci (Lond), 58 (1980) 21-27.

[52] C. Lerma, O. Infante, H. Perez-Grovas, M.V. Jose, Poincare plot indexes of heart rate variability capture dynamic adaptations after haemodialysis in chronic renal failure patients, Clin Physiol Funct I, 23 (2003) 72-80.

[53] S. Akselrod, D. Gordon, F.A. Ubel, D.C. Shannon, A.C. Barger, R.J. Cohen, Power Spectrum Analysis of Heart-Rate Fluctuation - a Quantitative Probe of Beat-to-Beat Cardiovascular Control, Science, 213 (1981) 220-222.

[54] P. Martínez-García, C. Lerma, O. Infante, Baroreflex sensitivity estimation by the sequence method with delayed signals, Clinical Autonomic Research, 22 (2012) 289-297.

[55] J. Gierałtowski, D. Hoyer, F. Tetschke, S. Nowack, U. Schneider, J. Żebrowski, Development of multiscale complexity and multifractality of fetal heart rate variability, Autonomic Neuroscience, 178 (2013) 29-36.

[56] P. Van Leeuwen, D. Cysarz, F. Edelhäuser, D. Grönemeyer, Heart rate variability in the individual fetus, Autonomic Neuroscience, 178 (2013) 24-28.

[57] T.N. James, Cardiac conduction system: fetal and postnatal development, The American journal of cardiology, 25 (1970) 213-226. 


\section{University Library}

\section{- M M N E R VA A gateway to Melbourne's research publications}

Minerva Access is the Institutional Repository of The University of Melbourne

Author/s:

Karmakar, C;Kimura, Y;Palaniswami, M;Khandoker, A

Title:

Analysis of fetal heart rate asymmetry before and after 35 weeks of gestation

Date:

2015-08-01

Citation:

Karmakar, C., Kimura, Y., Palaniswami, M. \& Khandoker, A. (2015). Analysis of fetal heart rate asymmetry before and after 35 weeks of gestation. BIOMEDICAL SIGNAL PROCESSING AND CONTROL, 21, pp.43-48. https://doi.org/10.1016/j.bspc.2015.05.010.

Persistent Link:

http://hdl.handle.net/11343/55371 